\title{
Email Accessibility and Social Networking
}

\author{
Brian Wentz and Jonathan Lazar \\ Department of Computer and Information Sciences, Center for Applied Information \\ Technology, and Universal Usability Laboratory, \\ Towson University 8000 York Road, Towson, MD 21252 \\ bwentz2@students.towson.edu, jlazar@towson.edu
}

\begin{abstract}
Previous studies concerning the accessibility of social networking web sites have revealed that there are components of such web sites which present accessibility problems for users with disabilities, including blind users. This paper discusses the intersection of e-mail accessible and social networking, for blind users. Not only is e-mail an important component of social networking sites, but often, an e-mail address is required for registration. The topic of e-mail and social networking is being studied in the broader context of a multi-stage research study of blind users and their e-mail usage. This multi-stage study is being conducted to understand the usage patterns and accessibility problems encountered by blind users in using both web-based and application-based e-mail. Our hope is that the research study will result in the proposal of new strategies and guidelines for accessible design. This conference presentation will report on the status of the data collection.
\end{abstract}

Keywords: social networking, blind users, email, web accessibility, CSCW.

\section{Introduction}

If a student at a college or university were asked today about the importance of social networking web sites such as MySpace or Facebook it would garner a response similar to questioning the importance of instant messaging or email only a few years ago. With $35 \%$ of adults and $65 \%$ of teens currently registered with social networking web sites [19], these online communities are undoubtedly having a significant impact on higher education. This impact could be positive, such as the ability to provide a sense of community to individuals who would either minimally or never form a social connection otherwise. The impact could also be negative, such as the immediate and global exposure of negative or illegal activities, or even the introduction of a new security threat as social networking web sites have become a target of viruses [20]. The registration for and use of social networking web sites has also been identified as a possible source for increased spam email [3].

The level of accessibility of social networking web sites for users with disabilities has been analyzed by several parties including the AbilityNet Web Accessibility Team [1]. The use of visual CAPTCHAs (random blurred characters that need to be entered for validation), poor support for keyboard-based navigation and the lack of 
alternate text for navigational elements were some of the problems discovered in social networking web sites [2]. Since email is highly integrated into social networking and also comprises a form of social networking [24], this paper will focus on the intersection between email accessibility for blind users and social networking.

\subsection{The Legal Importance of Web Accessibility}

Recent court cases regarding web accessibility in the United States may begin to motivate companies and web services to begin to adopt a more proactive approach to the challenge of electronic accessibility. While Section 508 of the Rehabilitation Act specifies accessibility standards for government web sites and technology, there are several other applicable mandates as well, including Section 504 and the E-government Act [8]. It is still unclear whether the law requires that private corporate web sites must be accessible. Recent legal rulings, although not final, point towards web accessibility being potentially covered under the Americans with Disabilities Act, which does cover private companies. Companies are encouraged to take a proactive approach to ensuring that their web sites can be used by the largest number of users [9]. A recent example of this is the court case involving Target.com and the National Federation of the Blind. In 2005, the National Federation of the Blind asked Target to correct its web site so that screen readers could use it more easily. In September 2008, Target settled the charges with the National Federation of the Blind, and the preliminary court rulings noted that the lack of accessibility on the web site could prevent blind users from sharing the same public accommodation of the physical store that others enjoy [27].

There are nearly 2 million individuals in the United States who are blind with no residual vision, and 37 million individuals worldwide who are blind with no residual vision [12][26]. This is a very large population of users, and technology should be accessible to anyone, regardless of background or physical abilities. This concept should follow through to include the accessibility and usability of technology for blind users [12], whether that technology is email or a social networking web site. It is the responsibility of the technology community to ensure that accessibility problems are not inherently occurring, and when accessibility problems are discovered, the effort must be extended to correct them.

\subsection{Social Networking Accessibility for Blind Users}

It is important for blind users to take part in social networking sites for multiple reasons. Social networking sites can be used to meet friends, catch up with long-lost acquaintances, and keep tabs on what friends are doing. Social networking sites can also be used to make business contacts. Web sites such as LinkedIn or ZoomInfo further facilitate social networking for professionals who need to develop business contacts for purposes such as job hunting [16]. Some individuals even use standard social networking sites for meeting potential dates [15].

The accessibility of social networking web sites (such as Facebook and MySpace) has been analyzed by AbilityNet in the U.K. One of the most critical accessibility flaws noted impacted blind users who access social networking web sites by using screen reader software (such as JAWS) [1]. This flaw is the use of CAPTCHAs to register for social networking web sites. Visual CAPTCHAs pose many problems for 
blind users. Both Facebook and MySpace require the use of a CAPTCHA during the registration process. MySpace offers only a visual CAPTCHA while Facebook also provides an audio CAPTCHA option [6][17]. AbilityNet found that other problems with social networking web sites could also impact users with disabilities, including the lack of skip links on pages, navigational graphics lacking alternate text (which is what the screen reader software uses in place of graphics), poor or misleading link text and no alternatives for features that use JavaScript [2]. The lack of alternate text for graphics or the use of graphics for navigational purposes has been a highlighted problem with web site accessibility in general. AbilityNet noted that social networking web sites have the opportunity to enable someone who has a disability to bridge the networking and socializing gap by removing some of the traditional physical barriers [1]. The World Wide Web Consortium also noted in its recent report on the future of social networking that many social networking websites have not yet even started to address accessibility requirements [25]. Technology, however, should be a tool to level the field of opportunity for all users, and as such it must be designed with the goal of accessibility for all in mind.

\subsection{The Interface between Email and Social Networking}

Email is one of the dominant components that form the core of many web-based applications and services. While it may not always be a public form of social networking, it is certainly a private aspect of social networking [8]. Companies such as Google and Yahoo have even discussed ways to turn their web-based email systems into a more enhanced social network. Google did not release specific details, but Yahoo described the changes as being email display based on relationships [24] since email is already a very personal form of social networking that provides contact with those who are socially the closest to an individual. One concrete example of the intersection of e-mail and social networking is party invitation web sites such as Evite [5]. Individuals receive an e-mail in their inbox, pointing them to a web site that lists information about an upcoming party that they are invited to. Usually, a web site like this includes information about the time and location of the party, a list of who has been invited, who has indicated that they are coming, and who has indicated that they will not be coming. After the party, information about what happened at the party and pictures and stories from the party are all posted on the web site. So to take part in this social network, not only must the web page with party information be accessible, but users must be able to receive and read the emails that point them to the web site. Since many emails are now sent with embedded HTML, emails that theoretically should be plain text and accessible may in fact not be accessible.

In addition to the private social networking aspect of email is the fact that email usage is actually required to even register (and use certain features of) social networking web sites such as Facebook or MySpace. The registration pages for both of these popular social networking web sites require a valid email address from which the email address book can later be used as a method of adding social networking contacts [6]. Email is therefore an inescapable facet of social networking.

\subsection{Email Accessibility for Blind Users}

Email is a primary means of communications and productivity in society today both in the workforce and on college and university campuses. It is often a simple way to 
share documents and quickly disseminate information. College and university students depend on email for private and public communication. It is therefore critical to know whether accessibility problems with email software are creating difficulties or even barriers for blind users.

Spam is a problem that can consume the time of both blind and sighted users, with almost two-thirds of email users citing spam as a major problem [12]. When the general annoyance and frustrations of spam is compounded with having the titles or portions of email messages being read to a user audibly through a screen reader, the level of embarrassment is likely to escalate given the fact that many spam emails contain inappropriate content. With spam filtering software representing the primary solution for preventing this unsolicited email, the ensuing problem is that a spam filter can still allow spam to reach a user and can also prevent legitimate email from reaching a user [4]. Spam is also a security issue since it is one of the most common venues for the distribution of viruses and worms [21]. The possibility that registration with a social networking web site may increase this growing problem could be a genuine point of concern for blind users.

Web-based email is also a problematic area for blind users. During a focus group for this study that was conducted in May 2008 (see details later in this paper), blind users noted that web-based email such as Yahoo Mail, Gmail and Hotmail all share the problems of cluttered audio interfaces, the use of visual CAPTCHAs (Gmail and Hotmail also provide audio CAPTCHAs [7][10]) and the lack of alternative text or use of poor alternate text for graphical navigational elements. These types of difficulties appear to be analogous to those that are faced by blind users who use social networking web sites. The lack of accessibility for most web-based email may discourage blind users from even attempting to use web-based email.

\section{Research on Email Accessibility for Blind Users}

A large, multi-stage research study is taking place to understand how blind users utilize e-mail, what challenges they face, and what improvements could be made.

The study is being conducted in collaboration with the National Federation of the Blind (hereafter referred to as NFB). The three stages of data collection are a focus group, a web-based survey, and the in-depth usability testing of e-mail software by blind users. The focus of the study is on blind users with no residual vision, who utilize screen readers, which are currently the primary tool of choice by blind users [18]. The topic of social networking and e-mail is one small facet of the study. This paper reports on the current status of the research study.

\subsection{Focus Group}

On May 1, 2008, a focus group of four employees of the NFB convened to evaluate the problem of email accessibility and to assist in the further definition of the information to be obtained in the second and third stages of the study. The group consisted of three males and one female, ranging in age from their mid-twenties to mid-fifties and the vocational background of the participants ranged from non-technical to technical in nature. While many of the focus group questions pertained to software-based email, the problems associated with web-based email were also discussed. 
The consensus of the focus group was that web-based email such as Yahoo Mail, Gmail and Hotmail were extremely cluttered when used with a screen reader. Additionally, simply signing up for a web-based email account proved to be difficult, since some providers require the use of a visual CAPTCHA during the registration process, which has been highlighted as a barrier to access [12]. The web-based navigational interfaces were also noted to be problematic. In particular, navigational elements that used graphics contained either poorly structured alternate text or no alternate text. Spam email messages were also noted to be a frustrating aspect of email, although NFB had recently implemented a very effective spam filtering system within its corporate email system, and the focus group participants reported it to be very effective. The users who used personal and web-based email reported that spam can be very frustrating and embarrassing, and their hope was that any spam filtering software used would work effectively without blocking any legitimate email.

\subsection{Web-Based Survey}

A web-based survey was developed as the second stage of the research project. The primary content of the survey was specifically based on the results and suggestions of the focus group. The survey content was also reviewed for suggestions by three additional researchers at Towson University. Before any electronic versions of the survey were created, the wording of the survey questions were checked for clarity, as is standard for electronic surveys [14]. Originally, a web-based survey tool called SurveyMonkey was used to develop the electronic version of the survey, due to its advertisement as a Section 508 compliant survey tool [23]. However, after testing, it was determined that the SurveyMonkey tool was in fact not entirely accessible using the JAWS screen reader software. The researchers attempted to work with SurveyMonkey technical support to correct the problem, but were unsuccessful. At this point, a different tool, SurveyGizmo, was used to develop the web-based version of the survey. Initial testing showed that SurveyGizmo did not have the same accessibility problems as SurveyMonkey. So the final online version of the web-based survey was created using SurveyGizmo [22]. The survey was then thoroughly tested for accessibility in JAWS versions 8 and 10 by the researchers, and it was also examined for usability and understandability by an independent blind user who is employed as an assistive technology specialist at the Pennsylvania Training and Technical Assistance Network. Multiple additional steps typically are needed when pre-testing an electronic survey tool for blind users [13].

Included in the survey are demographic questions such as age, gender, employment status and academic status. Questions regarding email usage frequency and estimated hours per day are also included. Since spam has been highlighted as a particular area of concern, questions regarding spam, and how it is dealt with, are asked as well. Special features of email such as contacts, calendaring and organization are also addressed, and there are separate sections on software-based and web-based email. Besides the focus on email usage and accessibility, this survey attempts to determine whether each participant is a student at a college or university and a social networking web site user. Because of this, questions were also included concerning the use of social networking web sites and any difficulties encountered when using social networking web sites. The survey is structured using skip logic so that if questions do not apply to a particular individual, those questions will not be asked. Example: if a 
survey respondent indicates that they do not use web-based e-mail, then they will not receive any follow-up questions about web-based e-mail.

\subsection{Current Status of Research}

The survey was officially released for responses in January 2009 through list servers sponsored by the Maryland and Pennsylvania chapters of the NFB and is still underway at the time of this writing. It is expected that by the time of the conference, complete results of the survey will be available for presentation. It is expected that the third stage of data collection, usability testing of different e-mail applications, will take place during the middle of 2009 . Some preliminary results from the usability testing may possibly be available by the time of the conference. The testing will be conducted in the natural work environment of the users, and user behavior will be observed during the use of software and web-based email applications using a screen reader. This stage will be more in depth and will involve each participant performing tasks such as using software and web-based email, organizing and retrieving email, and also using features such as the contacts and calendar. The purpose of this stage is to more fully understand the usage patterns, as well as any problems that are encountered.

\section{Summary}

There needs to be an increased amount of research into both the accessibility of social networking web sites, and understanding how blind users use e-mail for both workrelated and social-related communication. There are many potential benefits of accessible social networking web sites, both for social connections, and also for work-related networking.

Acknowledgments. The National Federation of the Blind has greatly assisted this research.

\section{References}

1. AbilityNet, http://www.abilitynet.co.uk/enation85

2. Accessites.org, http://accessites.org/site/2008/01/ social-networking-not-for-everyone

3. Brown, G., How, T., Ihbe, M., Prakash, A., Borders, K.: Social Networks and ContextAware Spam. In: CSCW 2008, pp. 403-412. ACM, New York (2008)

4. Cormack, G., Lynam, T.: Online Supervised Spam Filter Evaluation. ACM Trans. Inf. Syst. 25(3), 1-31 (2007)

5. Evite, http://www. evite.com

6. Facebook, http://www. facebook. com

7. Gmail, http://www.google.com

8. Guy, I., Jacovi, M., Meshulam, N., Ronen, I., Shahar, E.: Public vs. Private - Comparing Public Social Network Information with Email. In: CSCW 2008, pp. 393-402. ACM, New York (2008) 
9. Hocheiser, H., Lazar, J.: HCI and Societal Issues: A Framework for Engagement. International Journal of Human-Computer Interaction 23(3), 339-374 (2007)

10. Hotmail, http://mail.live.com/mail

11. Jaeger, P.: Beyond Section 508: The Spectrum of Legal Requirements for Accessible EGovernment Web Sites in the United States. Journal of Government Information 30(4), 518-533 (2004)

12. Lazar, J. (ed.): Universal Usability: Designing Computer Interfaces for Diverse Users. John Wiley \& Sons, Chichester (2007)

13. Lazar, J., Allen, A., Kleinman, J., Lawrence, J.: Methodological Issues in Using Time Diaries to Collect Frustration Data from Blind Computer Users. In: Proceedings of the $11^{\text {th }} \mathrm{In}-$ ternational Conference on Human-Computer Interaction. Proceedings on CD-ROM (2005)

14. Lazar, J., Preece, J.: Using Electronic Surveys to Evaluate Networked Resources: From Idea to Implementation. In: Evaluating Networked Information: Techniques, Policy, and Issues, pp. 137-154. Information Today, Medford (2001)

15. Lee, A., Bruckman, A.: Judging You by the Company You Keep: Dating on Social Networking Sites. In: Proceedings of the 2007 international ACM Conference on Supporting Group Work, pp. 371-378. ACM, New York (2007)

16. LinkedIn, http: //www. Iinkedin. com

17. MySpace, http://www. myspace.com

18. NFB, http://www.nfb.org/nfb/Louis_Braille_coin_facts.asp?SnID=2

19. Pew Internet \& American Life Project, http://www.pewinternet.org/PPF/r/272/report_display.asp

20. Reuters, http: / /www.reuters.com/article/newsOne/idUSTRE4B3 7LV2 0081204

21. Stolfo, S., Hershkop, S., Hu, C., Li, W., Nimeskern, O., Wang, K.: Behavior-Based Modeling and its Application to Email Analysis. ACM Trans. Inf. Tech. 6(2), 187-221 (2006)

22. SurveyGizmo, http: //www. surveygizmo.com

23. SurveyMonkey, http: //www. surveymonkey.com/HelpCenter/ Answer . aspx? HelpID $=247$

24. The New York Times, http: //bits.blogs.nytimes.com/2007/11/13/ inbox-20-yahoo-and-google-to-turn-e-mail-into-a-social-network

25. W3C, http://www.w3 .org/2008/09/msnws/report

26. World Health Organization, http: / / www. who. int/mediacentre/factsheets/fs282/en

27. Whitfield, N.: Target Settles Case over Web Site Access for the Blind. Business Insurance 42(36), 4, 40 (2008) 